\title{
Effects of standardized ileal digestible tryptophan: lysine ratio on growth performance of nursery pigs ${ }^{1,2}$
}

\author{
M. A. D. Gonçalves, * S. Nitikanchana,* M. D. Tokach, $\uparrow$ S. S. Dritz, ${ }^{3}$ N. M. Bello, † \\ R. D. Goodband, $\uparrow$ K. J. Touchette, § J. L. Usry,§ J. M. DeRouchey, $\uparrow$ and J. C. Woodworth $\uparrow$ \\ *Department of Diagnostic Medicine/Pathobiology, College of Veterinary Medicine, \\ Kansas State University, Manhattan 66506-0201; $†$ Department of Animal Sciences and Industry, College \\ of Agriculture, Kansas State University, Manhattan 66506-0201; \$Department of Statistics, College of Arts \\ and Sciences, Kansas State University, Manhattan 66506-0201; and §Ajinomoto Heartland Inc., Chicago, IL 60631
}

\begin{abstract}
Two experiments were conducted to estimate the standardized ileal digestible (SID) Trp:Lys ratio requirement for growth performance of nursery pigs. Experimental diets were formulated to ensure that lysine was the second limiting AA throughout the experiments. In Exp. 1 (6 to $10 \mathrm{~kg} \mathrm{BW),} 255$ nursery pigs (PIC $327 \times 1050$, initially $6.3 \pm 0.15 \mathrm{~kg}$, mean \pm SD) arranged in pens of 6 or 7 pigs were blocked by pen weight and assigned to experimental diets ( 7 pens/ diet) consisting of SID Trp:Lys ratios of $14.7 \%, 16.5 \%$, $18.4 \%, 20.3 \%, 22.1 \%$, and $24.0 \%$ for $14 \mathrm{~d}$ with $1.30 \%$ SID Lys. In Exp. 2 (11 to $20 \mathrm{~kg} \mathrm{BW),} \mathrm{1,088} \mathrm{pigs} \mathrm{(PIC}$ $337 \times 1050$, initially $11.2 \mathrm{~kg} \pm 1.35 \mathrm{BW}$, mean $\pm \mathrm{SD}$ ) arranged in pens of 24 to 27 pigs were blocked by average pig weight and assigned to experimental diets (6 pens/diet) consisting of SID Trp:Lys ratios of 14.5\%, $16.5 \%, 18.0 \%, 19.5 \%, 21.0 \%, 22.5 \%$, and $24.5 \%$ for 21 d with $30 \%$ dried distillers grains with solubles and $0.97 \%$ SID Lys. Each experiment was analyzed using general linear mixed models with heterogeneous residual variances. Competing heteroskedastic models included broken-line linear (BLL), broken-line quadratic (BLQ), and quadratic polynomial (QP). For each response, the best-fitting model was selected using
\end{abstract}

Bayesian information criterion. In Exp. 1 (6 to $10 \mathrm{~kg}$ BW), increasing SID Trp:Lys ratio linearly increased $(P<0.05)$ ADG and G:F. For ADG, the best-fitting model was a QP in which the maximum ADG was estimated at $23.9 \%(95 \%$ confidence interval [CI]: $[<14.7 \%,>24.0 \%])$ SID Trp:Lys ratio. For G:F, the best-fitting model was a BLL in which the maximum G:F was estimated at 20.4\% (95\% CI: [14.3\%, 26.5\%]) SID Trp:Lys. In Exp. 2 (11 to $20 \mathrm{~kg} \mathrm{BW}$ ), increasing SID Trp:Lys ratio increased $(P<0.05)$ ADG and G:F in a quadratic manner. For ADG, the best-fitting model was a QP in which the maximum ADG was estimated at $21.2 \%$ (95\% CI: $[20.5 \%, 21.9 \%])$ SID Trp:Lys. For G:F, BLL and BLQ models had comparable fit and estimated SID Trp:Lys requirements at $16.6 \%(95 \%$ CI: $[16.0 \%, 17.3 \%])$ and $17.1 \%(95 \% \mathrm{CI}:[16.6 \%$, $17.7 \%])$, respectively. In conclusion, the estimated SID Trp:Lys requirement in Exp. 1 ranged from $20.4 \%$ for maximum G:F to $23.9 \%$ for maximum ADG, whereas in Exp. 2 it ranged from $16.6 \%$ for maximum G:F to $21.2 \%$ for maximum ADG. These results suggest that standard NRC (2012) recommendations may underestimate the SID Trp:Lys requirement for nursery pigs from 11 to $20 \mathrm{~kg} \mathrm{BW}$.

Key words: amino acid ratio, growth, lysine, nursery pig, tryptophan

(C) 2015 American Society of Animal Science. All rights reserved.

\footnotetext{
${ }^{1}$ Contribution no. 15-325-J from the Kansas Agricultural Experiment Station (Manhattan, KS).

${ }^{2}$ Appreciation is expressed to New Horizon Farms (Pipestone, $\mathrm{MN}$ ) for providing animals and research facilities and to A. Morris, C. Steck, and M. Heintz for technical assistance.

${ }^{3}$ Corresponding author: dritz@k-state.edu

Received March 8, 2015.

Accepted May 25, 2015.
}

J. Anim. Sci. 2015.93:3909-3918 doi:10.2527/jas2015-9083

\section{INTRODUCTION}

Tryptophan is an important limiting AA in corn and soybean meal-based diets of nursery and finishing pigs (Lewis, 2000). As the availability of feedgrade AA, including Trp, increases, so does their use as replacement for intact protein sources in swine diets. The Trp requirement in swine diets can be 
expressed in different ways. In particular, the standardized ileal digestible (SID) Trp requirement expressed as a ratio to Lys (Trp:Lys) is considered a practical approach for diet formulation (Stein et al., 2007); however, the observed SID Trp:Lys ratio requirement varies considerably among studies. For example, NRC (2012) estimates the SID Trp requirement at $16.3 \%$ of Lys for nursery pigs, but Guzik et al. (2005) estimated a SID Trp:Lys requirement of greater than $19.5 \%$, and Simongiovanni et al. (2012) concluded that it was $17 \%$ to $22 \%$ of Lys. The objective of these studies was to estimate the SID Trp:Lys ratio requirement for growth performance in nursery pigs.

\section{MATERIALS AND METHODS}

The Kansas State University Institutional Animal Care and Use Committee approved the protocols used in these experiments. Experiment 1 was conducted at the Kansas State University Swine Teaching and Research Center in Manhattan, and Exp. 2 was conducted at a commercial research-nursery barn in southwestern Minnesota.

\section{Experiment 1: 6 to $10 \mathrm{~kg} \mathrm{BW}$}

A total of 255 nursery pigs (PIC $327 \times 1050$, with initial and final BW of $6.3 \pm 0.15$ and $9.8 \pm 0.46 \mathrm{~kg}$, respectively, mean $\pm \mathrm{SD}$ ) were used in a 14-d growth trial. Pigs were weaned at $21 \mathrm{~d}$ of age and placed in the nursery facility, where they were fed a common diet for $3 \mathrm{~d}$. At d 3 after weaning, pigs were weighed in pens, and pens were randomly assigned to dietary treatments in a randomized complete block design blocked by initial average pen BW. Therefore, d 3 after weaning was d 0 of the trial. Each treatment consisted of 7 pens of 6 to 7 pigs/pen, and each pen comprised barrows and gilts. A 4-hole, dry self-feeder and a nipple waterer were used in each pen $(1.2 \times 1.5 \mathrm{~m})$ to provide ad libitum access to feed and water. Experimental diets consisted of corn and soybean meal and had 6 ratios of SID Trp:Lys, namely, 14.7\%, 16.5\%, 18.4\%, 20.3\%, 22.1\%, and $24.0 \%$. Feed-grade L-Trp was added at the expense of corn starch in the basal diet to achieve the desired ratios of SID Trp:Lys (Table 1). Nutrients and SID AA digestibility values used for diet formulation were obtained from NRC (1998). Large batches of the 14.7\% and $24.0 \%$ SID Trp:Lys diets were manufactured, then blended to achieve the intermediate SID Trp:Lys ratios. The percentages of low- and high-SID Trp:Lys blends to create the treatment diets were $100 \%$ and $0 \%, 80 \%$ and $20 \%, 60 \%$ and $40 \%, 40 \%$ and $60 \%, 20 \%$ and $80 \%$, and $0 \%$ and $100 \%$ for $14.7 \%, 16.5 \%, 18.4 \%, 20.3 \%, 22.1 \%$, and $24.0 \%$ SID Trp:Lys, respectively. Diets were
Table 1. Diet composition, Exp. 1 (as-fed basis) ${ }^{1}$

\begin{tabular}{|c|c|}
\hline Item & Basal diet \\
\hline \multicolumn{2}{|l|}{ Ingredient, $\%$} \\
\hline Corn & 58.09 \\
\hline Soybean meal $(46.5 \% \mathrm{CP})$ & 25.20 \\
\hline Spray-dried whey & 10.00 \\
\hline Soybean oil & 1.00 \\
\hline Monocalcium P (21\% P) & 1.10 \\
\hline Limestone & 0.90 \\
\hline Salt & 0.35 \\
\hline Zinc oxide & 0.25 \\
\hline Trace mineral premix ${ }^{2}$ & 0.15 \\
\hline Vitamin premix ${ }^{3}$ & 0.25 \\
\hline L-Lys $\mathrm{HCl}$ & 0.533 \\
\hline DL-Met & 0.220 \\
\hline L-Thr & 0.230 \\
\hline L-Ile & 0.100 \\
\hline L-Val & 0.160 \\
\hline Gln & 0.630 \\
\hline Gly & 0.630 \\
\hline Phytase $^{4}$ & 0.085 \\
\hline Corn starch ${ }^{5}$ & 0.123 \\
\hline L-Trp & - \\
\hline Total & 100 \\
\hline \multicolumn{2}{|l|}{ Calculated analysis } \\
\hline \multicolumn{2}{|l|}{ SID AA, ${ }^{6} \%$} \\
\hline Lys & 1.30 \\
\hline Ile:Lys & 60 \\
\hline Leu:Lys & 111 \\
\hline Met:Lys & 36 \\
\hline Met and Cys:Lys & 58 \\
\hline Thr:Lys & 64 \\
\hline Trp:Lys & 14.7 \\
\hline Val:Lys & 70 \\
\hline Total Lys, $\%$ & 1.42 \\
\hline $\mathrm{ME}, \mathrm{kcal} / \mathrm{kg}$ & 3,341 \\
\hline $\mathrm{NE}, \mathrm{kcal} / \mathrm{kg}$ & 2,239 \\
\hline SID lysine:ME, g/Mcal & 3.89 \\
\hline SID lysine:NE, g/Mcal & 5.27 \\
\hline $\mathrm{CP}, \%$ & 20.4 \\
\hline $\mathrm{Ca}, \%$ & 0.72 \\
\hline $\mathrm{P}, \%$ & 0.64 \\
\hline Available P, \% & 0.47 \\
\hline
\end{tabular}

${ }^{1}$ Diets were fed from 6.8 to $9.8 \mathrm{~kg} \mathrm{BW}$. Nutrient content was calculated on the basis of values from NRC (1998).

${ }^{2}$ Provided per kilogram of diet: $39.6 \mathrm{mg}$ Mn from manganese oxide, $165 \mathrm{mg}$ Fe from iron sulfate, $1,965 \mathrm{mg} \mathrm{Zn}$ from zinc sulfate, $16.5 \mathrm{mg} \mathrm{Cu}$ from copper sulfate, $0.30 \mathrm{mg}$ I from calcium iodate, and $0.30 \mathrm{mg}$ Se from sodium selenite.

${ }^{3}$ Provided per kilogram of diet: 11,020 IU vitamin A, 1,378 IU vitamin $\mathrm{D}_{3}$, $44 \mathrm{IU}$ vitamin $\mathrm{E}, 4 \mathrm{mg}$ vitamin $\mathrm{K}, 8 \mathrm{mg}$ riboflavin, $28 \mathrm{mg}$ pantothenic acid, $50 \mathrm{mg}$ niacin, and $0.039 \mathrm{mg}$ vitamin $\mathrm{B}_{12}$.

${ }^{4}$ Phyzyme 600 (Danisco Animal Nutrition, St. Louis, MO) provided 509 phytase activity ( FTU) per kilogram of diet, with a release of $0.10 \%$ available $P$.

${ }^{5}$ Feed-grade L-Trp was added at the expense of corn starch at $0 \%$, $0.024 \%, 0.049 \%, 0.074 \%, 0.098 \%$, and $0.123 \%$ of the diet to provide Trp:Lys ratios of $14.7 \%, 16.5 \%, 18.4 \%, 20.3 \%, 22.1 \%$, and $24.0 \%$ to form the experimental treatments.

${ }^{6} \mathrm{SID}=$ standardized ileal digestible. 
formulated to $1.30 \%$ SID Lys on the basis of data of Nemechek et al. (2011). On the basis of the NRC (2012) model, 1.34\% SID Lys is the requirement in a diet with $3,341 \mathrm{kcal} \mathrm{ME} / \mathrm{kg}$ for $10-\mathrm{kg}$ nursery pigs. Thus, experimental diets were $0.04 \%$ below the NRC requirement at the end of the 6- to $10-\mathrm{kg}$ BW nursery phase to ensure that lysine was the second limiting AA throughout the experiment. The $14.7 \%$ SID Trp:Lys ratio diet was also reported to be deficient in Trp (Nemechek et al., 2011). Diets contained $10 \%$ spray-dried whey and no specialty protein sources, such as spray-dried blood meal or select menhaden fish meal. Experimental diets were fed in meal form and were prepared at the O. H. Kruse Feed Technology Innovation Center in Manhattan, KS.

\section{Experiment 2: 11 to $20 \mathrm{~kg} \mathrm{BW}$}

A total of 1,088 pigs (PIC $337 \times 1050$, with initial and final BW of $11.2 \mathrm{~kg} \pm 1.35$ and $20.3 \pm 2.16 \mathrm{~kg}$, respectively, mean $\pm \mathrm{SD}$ ) were used in a 21 -d growth trial. Pigs were weaned at $16 \mathrm{~d}$ of age and grouped into pens of 27 animals (14 gilts and 13 barrows). After weaning, pigs were fed a common pelleted diet for $7 \mathrm{~d}$, followed by common meal diets containing $10 \%$ and $20 \%$ dried distillers grains with solubles (DDGS) from $\mathrm{d} 7$ to 14 and 14 to 28 after weaning, respectively, with $20 \%$ SID Trp:Lys ratio. On d 28 after weaning, pigs were weighed in pens, and pens were blocked by initial average pen BW and randomly assigned dietary treatments in a randomized complete block design with 6 pens per treatment. Therefore, d 28 after weaning was $\mathrm{d} 0$ of the trial. The facility was totally enclosed, environmentally controlled, and mechanically ventilated. Pens had completely slatted flooring and deep pits for manure storage. Each pen $(3.7 \times 2.3 \mathrm{~m})$ was equipped with a 6 -hole stainless steel dry self-feeder and a pan waterer for ad libitum access to feed and water. Daily feed additions to each pen were accomplished through a robotic feeding system (FeedPro, Feedlogic Corp., Willmar, MN) capable of providing and measuring feed amounts for individual pens. Five representative samples of corn, soybean meal, and DDGS were collected each week for $5 \mathrm{wk}$ and were analyzed in duplicate for total AA (method 994.12; AOAC International, 2012) and CP (method 990.03; AOAC International, 2012) by Ajinomoto Heartland Inc. (Chicago, IL) before diet formulation. These values along with standardized digestibility coefficients from NRC (2012) for corn, soybean meal, and DDGS were used in diet formulation. Diets were balanced on an NE basis using NRC (2012) values.

Two experimental corn-soybean meal-based diets with $30 \%$ DDGS were formulated (Table 2) to be limiting in Lys and to have $14.5 \%$ and $24.5 \%$ SID Trp:Lys ratios; then the diets were blended using the robotic
Table 2. Diet composition, Exp. 2 (as-fed basis) ${ }^{1}$

\begin{tabular}{|c|c|c|}
\hline \multirow[b]{2}{*}{ Item } & \multicolumn{2}{|c|}{ SID Trp:Lys } \\
\hline & Low $(14.5 \%)$ & $\operatorname{High}(24.5 \%)$ \\
\hline \multicolumn{3}{|l|}{ Ingredient, \% } \\
\hline Corn & 55.15 & 55.05 \\
\hline Soybean meal (46\% CP) & 10.91 & 10.92 \\
\hline DDGS & 30.00 & 30.00 \\
\hline Beef tallow & 0.50 & 0.50 \\
\hline Dicalcium phosphate $(18.5 \% \mathrm{P})$ & 0.50 & 0.50 \\
\hline Limestone & 1.48 & 1.48 \\
\hline Salt & 0.35 & 0.35 \\
\hline Trace mineral premix ${ }^{2}$ & 0.100 & 0.100 \\
\hline Vitamin premix ${ }^{3}$ & 0.125 & 0.125 \\
\hline L-Lys HCL & 0.575 & 0.575 \\
\hline DL-Met & 0.070 & 0.070 \\
\hline L-Thr & 0.140 & 0.140 \\
\hline L-Trp & - & 0.098 \\
\hline L-Ile & 0.010 & 0.010 \\
\hline L-Val & 0.060 & 0.060 \\
\hline Phytase $^{4}$ & 0.025 & 0.025 \\
\hline Total & 100 & 100 \\
\hline \multicolumn{3}{|l|}{ Calculated analysis } \\
\hline \multicolumn{3}{|l|}{ SID AA, \% } \\
\hline Lys & 0.97 & 0.97 \\
\hline Ile:Lys & 55 & 55 \\
\hline Leu:Lys & 153 & 153 \\
\hline Met:Lys & 35 & 35 \\
\hline Met and Cys:Lys & 60 & 60 \\
\hline Thr:Lys & 65 & 65 \\
\hline Trp:Lys & 14.5 & 24.5 \\
\hline Val:Lys & 70 & 70 \\
\hline His:Lys & 38 & 38 \\
\hline Phe and Tyr:Lys & 106 & 106 \\
\hline Trp:BCAA ${ }^{5}$ & 3.9 & 6.6 \\
\hline Trp:LNAA ${ }^{6}$ & 2.8 & 4.8 \\
\hline $\mathrm{ME}, \mathrm{kcal} / \mathrm{kg}$ & 3,325 & 3,328 \\
\hline $\mathrm{NE}, \mathrm{kcal} / \mathrm{kg}$ & 2,466 & 2,468 \\
\hline SID lysine:ME, g/Mcal & 2.91 & 2.91 \\
\hline SID lysine:NE, g/Mcal & 3.93 & 3.93 \\
\hline $\mathrm{CP}, \%$ & 18.1 & 18.2 \\
\hline $\mathrm{Ca}, \%$ & 0.71 & 0.71 \\
\hline $\mathrm{P}, \%$ & 0.49 & 0.49 \\
\hline Available P, \% & 0.40 & 0.40 \\
\hline
\end{tabular}

${ }^{1}$ Diets were fed from 11.2 to $20.3 \mathrm{~kg} \mathrm{BW}$. Corn, dried distillers grains with solubles (DDGS), and soybean meal were analyzed for $\mathrm{CP}$ and total AA content, and NRC (2012) standardized ileal digestible (SID) digestibility values were used in the diet formulation.

${ }^{2}$ Provided per kilogram of diet: $33 \mathrm{mg}$ Mn from manganese oxide, 110 $\mathrm{mg}$ Fe from iron sulfate, $110 \mathrm{mg} \mathrm{Zn}$ from zinc oxide, $16.5 \mathrm{mg} \mathrm{Cu}$ from copper sulfate, $0.33 \mathrm{mg}$ I from ethylenediamin dihydroiodide, and $0.30 \mathrm{mg}$ Se from sodium selenite.

${ }^{3}$ Provided per kilogram of diet: 5,290 IU vitamin A, $827 \mathrm{IU}$ vitamin $\mathrm{D}_{3}$, $26.4 \mathrm{IU}$ vitamin $\mathrm{E}, 2.64 \mathrm{mg}$ vitamin $\mathrm{K}, 16.5 \mathrm{mg}$ pantothenic acid, $30 \mathrm{mg}$ niacin, $4.6 \mathrm{mg}$ riboflavin, and $0.02 \mathrm{mg}$ vitamin $\mathrm{B}_{12}$.

${ }^{4}$ OptiPhos 2000 (Enzyvia LLC, Sheridan, IN) provided 1,251 phytase activity (FTU) per kilogram of diet with a release of $0.13 \%$ available $P$.

${ }^{5}$ Amount of Trp in the diet as a ratio to branched-chain AA (BCAA; Ile, Leu, and Val) on an SID basis.

${ }^{6}$ Amount of Trp in the diet as a ratio to large neutral AA (LNAA; Ile, Leu, Val, Phe, and Tyr) on an SID basis. 
feeding system to achieve intermediate SID Trp:Lys ratios, thereby defining dietary treatments. The percentages of low- and high-SID Trp:Lys ratios blended to create the treatment diets were $100 \%$ and $0 \%, 80 \%$ and $20 \%, 65 \%$ and $35 \%, 50 \%$ and $50 \%, 35 \%$ and $65 \%$, $20 \%$ and $80 \%$, and $0 \%$ and $100 \%$ for $14.5 \%, 16.5 \%$, $18.0 \%, 19.5 \%, 21.0 \%, 22.5 \%$, and $24.5 \%$ SID Trp:Lys, respectively. The SID Trp:Lys ratio was increased by adding feed-grade L-Trp to the control diet at the expense of corn. The NRC (2012) model was used to estimate the SID Lys requirement of pigs fed diets with $2,466 \mathrm{kcal} \mathrm{NE} / \mathrm{kg}$ at the expected BW at the end of the experiment $(22.7 \mathrm{~kg})$. The SID Lys requirement (i.e., $1.07 \%$ ) was reduced by $0.10 \%$ point for diet formulation to ensure that lysine was the second limiting AA throughout the experiment. Diets were fed in meal form and were manufactured at the New Horizon Farms Feed Mill (Pipestone, MN). A preliminary experiment was conducted before Exp. 2 in the same facility and with pigs of the same BW to validate that diets were indeed limiting in Lys. For that preliminary experiment, a total of 1,188 pigs (PIC $337 \times 1050$, initially $12.9 \mathrm{~kg} \pm 0.66 \mathrm{BW}$, mean $\pm \mathrm{SD}$ ) were used in a 21-d growth trial with 27 pigs per pen and 11 pens per treatment. Pigs were fed either a high-Lys diet (SID Lys levels were $0.01 \%$ above the estimated NRC [2012] requirement at the expected initial BW) or a low-Lys diet $(0.97 \%$, which is $0.10 \%$ below the estimated NRC [2012] requirement at the expected final $\mathrm{BW})$. In the preliminary study, pigs fed low-Lys diets had lower $(P<0.05)$ ADG, ADFI, and G:F compared with pigs fed high-Lys diets, thus validating the below-requirement SID Lys level of diets used in Exp. 2.

\section{Diet Sampling and Analysis}

In Exp. 1 and 2, samples of the diets were submitted to Ward Laboratories Inc. (Kearney, NE) for analysis of DM (method 935.29; AOAC International, 2012), CF (method 978.10 [AOAC International, 2012] for preparation and Ankom 2000 Fiber Analyzer, Ankom Technology, Fairport, NY), ash (method 942.05; AOAC International, 2012), crude fat (method 920.39a [AOAC International 2012] for preparation and ANKOM XT20 Fat Analyzer, Ankom Technology), $\mathrm{Ca}$, and $\mathrm{P}$ (method 968.08b [AOAC International, 2012] for preparation using ICAP 6500, ThermoElectron Corp., Waltham, MA). In Exp. 1, CP was analyzed by Ward Laboratories Inc. (method 990.03; AOAC International, 2012); in Exp. 2, CP was analyzed by Ajinomoto Heartland Inc. (method 990.03; AOAC International, 2012).

In Exp. 1, diet samples were collected from feeders at the beginning of the trial and on $\mathrm{d} 7$ and 14. At the end of the trial, samples of the diets were combined within dietary treatment, and a composite sample from each treatment was analyzed in duplicate for total AA content by Ajinomoto Heartland Inc.

In Exp. 2, diet samples were taken from 6 feeders per dietary treatment $3 \mathrm{~d}$ after the beginning of the trial and $3 \mathrm{~d}$ before the end of the trial and stored at $-20^{\circ} \mathrm{C}$; then $\mathrm{CP}$ and total AA analyses were conducted in duplicate on composite samples by Ajinomoto Heartland Inc.

\section{Data Collection}

Pig BW and feed disappearance were measured on d 0 and 14 in Exp. 1 and on $d 0$ and 21 in Exp. 2 to calculate ADG, ADFI, G:F, grams of SID Trp intake per day, and grams of SID Trp intake per kilogram of gain. Total grams of SID Trp intake per day were calculated on the basis of formulated values by multiplying ADFI by SID Lys level by SID Trp:Lys. The total grams of SID Trp intake were divided by total BW gain to calculate the grams of SID Trp intake per kilogram of gain.

\section{Statistical Analysis}

Responses of interest (ADG, ADFI, G:F, BW, grams of SID Trp intake per day, and grams of SID Trp intake per kilogram of gain) measured at the pen level were analyzed using general linear and nonlinear mixed models to accommodate the randomized complete block design of the study. The linear predictor included the fixed effect of dietary treatment presented as a factor and initial average pen BW as a random blocking factor. Pen was the experimental unit. Models were expanded to account for heterogeneous residual variances, as needed. Residual assumptions were checked using standard diagnostics on Studentized residuals and were found to be reasonably met. Linear and quadratic polynomial contrasts were built to evaluate the functional form of the dose response to increasing the dietary SID Trp:Lys ratio on ADG, ADFI, G:F, BW, grams of SID Trp intake per day, and grams of SID Trp intake per kilogram of gain. Polynomial contrast coefficients were adjusted for unequally spaced treatments using the IML procedure of SAS (version 9.3; SAS Inst. Inc., Cary, NC). Degrees of freedom were estimated using the Kenward-Rogers approach. Statistical models were fitted using the GLIMMIX procedure of SAS. Results were considered significant at $P \leq 0.05$ and marginally significant at $0.05<P \leq 0.10$.

Additional models adapted from Robbins et al. (2006) and Pesti et al. (2009) were fitted to ADG and G:F to further estimate SID Trp:Lys requirements using an inverse prediction strategy. Specifically, competing statistical models fitted to the data included a broken-line linear 
ascending (BLL) model, a broken-line quadratic ascending (BLQ) model, and a quadratic polynomial (QP) model. As in Robbins et al. (2006), competing models described growth performance as a function of SID Trp:Lys levels as follows: For the BLL ascending model,

$$
\begin{array}{ll}
y_{i j}=L_{B L L}+U_{l}\left(R_{B L L}-X_{j}\right)+b_{j}+e_{i j} & \text { for } X_{i}<R_{B L L}, \\
y_{i j}=L_{B L L}+b_{j}+e_{i j} & \text { for } X_{i} \geq R_{B L L} .
\end{array}
$$

For the BLQ ascending model,

$$
\begin{array}{ll}
y_{i j}=L_{B L Q}+U_{q}\left(R_{B L Q}-X_{i}\right)^{2}+b_{j}+e_{i j} & \text { for } X_{i}<R_{B L Q,}, \\
y_{i j}=L_{B L Q}+b_{j}+e^{i j} & \text { for } X_{i} \geq R_{B L Q} .
\end{array}
$$

For the QP model,

$$
y_{i j}=\beta_{0}+\beta_{1} X_{i}+\beta_{2} X_{i}^{2}+b_{j}+e_{i j} .
$$

Here $y_{i j}$ is the response associated with the pen in block $j$ assigned to dietary treatment $i, X_{i}$ is the SID Trp:Lys level of the $i$ th dietary treatment, and $L_{\mathrm{BLL}}$ and $L_{\mathrm{BLQ}}$ indicate the unknown maximum growth response to dietary treatments (i.e., plateau) under the BLL and BLQ models, respectively. $R_{B L L}$ and $R_{B L Q}$ are the unknown minimum levels of SID Trp:Lys required to reach the plateau under the BLL and BLQ models, respectively. In the last equation, $\beta_{0}$ is the intercept; $U_{l} U_{q}, \beta_{1}$, and $\beta_{2}$ are the corresponding unknown rates of change of the response as a function of $X_{i} ; b_{j}$ is the random blocking effect of initial average pen BW associated with $j$ th block, where $b_{j} \sim N\left(0, \sigma_{b}^{2}\right)$; and $e_{i j}$ is a random error associated with the pen in the $j$ th block that received the $i$ th treatment, where $e_{i j} \sim N\left(0, \sigma_{e_{i}}^{2}\right)$. Note that $b_{j}$ and $e_{i j}$ are assumed to be independent of each other.

Broken-line regression models were fitted using the NLMIXED procedures of SAS. The optimization technique used was the dual quasi-Newton algorithm, as specified by default in the NLMIXED procedure. Competing statistical models were compared using maximum likelihood-based fit criteria, specifically the Schwarz Bayesian information criterion (BIC; Milliken and Johnson, 2009). Results reported here correspond to inference yielded by the best-fitting models.

For the best-fitting models, the estimated requirement of SID Trp:Lys to reach plateau performance (i.e., $R_{B L L}$ and $R_{B L Q}$ in the broken-line models) or to reach maximum performance (i.e., in the $\mathrm{QP}$ ) of $\mathrm{ADG}$ and $\mathrm{G}: \mathrm{F}$ are reported with a $95 \%$ confidence interval
(CI). In the quadratic polynomial model, the level of the SID Trp:Lys ratio that maximized ADG and G:F was estimated by equating the first derivative of the regression equation to zero, then solving for the SID Trp:Lys ratio (Pesti et al., 2009). The corresponding 95\% CI were computed using the inverse regression approach proposed by Lavagnini and Magno (2006).

\section{RESULTS AND DISCUSSION}

The analyzed total AA, DM, CP, CF, Ca, P, fat, and ash contents of diets for Exp. 1 and 2 (Tables 3 and 4 , respectively) were consistent with calculated values based on variation reported by Cromwell et al. (1999).

In Exp. 1 (6 to $10 \mathrm{~kg} \mathrm{BW}$ ), increasing the SID Trp:Lys ratio linearly increased (Table 5) ADG $(P=$ 0.022), G:F $(P=0.012)$, grams of SID Trp intake per day $(P=0.001)$, and grams of SID Trp intake per kilogram of gain $(P=0.001)$. Increasing the SID Trp:Lys ratio also induced marginal linear increases in ADFI $(P=0.057)$ and final BW $(P=0.052)$.

For ADG in the 6- to 10-kg BW pigs (Exp. 1), the best-fitting model was a QP (BIC: 215.3) compared with BLL and BLQ models (BIC: 217.4 and 217.8, respectively). The estimated regression equation for the best-fitting QP model (Fig. 1) was

$$
\mathrm{ADG}=42.7+1,819.7(\text { Trp:Lys })-3,810.1(\text { Trp:Lys })^{2}
$$

whereby the SID Trp:Lys explanatory variable is expressed as a proportion (i.e., 0.180) rather than a percentage (i.e., 18.0\%) for numerical stability of computations. Based on the best-fitting QP model, the maximum ADG was estimated at a $23.9 \%(95 \%$ CI: $[<14.7 \%$, $>24.0 \%]$ ) SID Trp:Lys ratio. Note the substantial width of this CI, thereby indicating considerable uncertainty for inference on SID Trp:Lys requirements that maximized ADG. This uncertainty is probably related to the large amount of unaccounted variability in ADG relative to an apparently minor effect of SID Trp:Lys ratios on ADG in Exp. 1, which is further supported by the nonsignificant $P$-value of the quadratic regression coefficient for $\mathrm{ADG}(P=0.484)$ during this phase.

Also for Exp. 1 (6 to $10 \mathrm{~kg} \mathrm{BW}$ ), the best-fitting model for G:F was a BLL (BIC: 253.6) compared with QP and BLQ (BIC: 255.0 and 255.0, respectively). The best-fitting estimated regression equations for the best-fitting BLL model (Fig. 2) were

$$
\begin{aligned}
\mathrm{G}: \mathrm{F}=0.733-0.6034 \times & (0.204-\text { Trp:Lys }) \\
& \text { if SID Trp:Lys }<20.4 \%
\end{aligned}
$$

$\mathrm{G}: \mathrm{F}=0.733$ if SID Trp:Lys $\geq 20.4 \%$ 
Table 3. Chemical analysis of the diets, Exp. 1 (as-fed basis) ${ }^{1}$

\begin{tabular}{|c|c|c|c|c|c|c|}
\hline \multirow[b]{2}{*}{ Item } & \multicolumn{6}{|c|}{ Standardized ileal digestible Trp:Lys ${ }^{2}$} \\
\hline & $14.7 \%$ & $16.5 \%$ & $18.4 \%$ & $20.3 \%$ & $22.1 \%$ & $24.0 \%$ \\
\hline \multicolumn{7}{|c|}{ Proximate analysis, $\%$} \\
\hline $\mathrm{DM}$ & $91.07(89.43)$ & $91.06(89.43)$ & $91.23(89.43)$ & $91.12(89.43)$ & $91.17(89.43)$ & $91.21(89.43)$ \\
\hline $\mathrm{CP}$ & $20.2(20.4)$ & $20.5(20.4)$ & $20.3(20.4)$ & $20.4(20.4)$ & $20.6(20.5)$ & $21.0(20.5)$ \\
\hline $\mathrm{CF}$ & $2.2(2.3)$ & $2.2(2.3)$ & $2.3(2.3)$ & $2.3(2.3)$ & $2.4(2.3)$ & $2.3(2.3)$ \\
\hline $\mathrm{Ca}$ & $0.87(0.72)$ & $0.85(0.72)$ & $0.76(0.72)$ & $0.84(0.72)$ & $0.86(0.72)$ & $0.87(0.72)$ \\
\hline $\mathrm{P}$ & $0.69(0.64)$ & $0.63(0.64)$ & $0.67(0.64)$ & $0.70(0.64)$ & $0.73(0.64)$ & $0.77(0.64)$ \\
\hline Fat & $3.3(3.7)$ & $3.2(3.7)$ & $3.4(3.7)$ & $3.1(3.7)$ & $3.2(3.7)$ & $3.2(3.7)$ \\
\hline Ash & $6.04(5.54)$ & $5.93(5.54)$ & $5.71(5.54)$ & $5.81(5.54)$ & $6.10(5.54)$ & $6.11(5.54)$ \\
\hline \multicolumn{7}{|l|}{ AA, $\%$} \\
\hline Lys & $1.43(1.42)$ & $1.43(1.42)$ & $1.42(1.42)$ & $1.38(1.42)$ & $1.37(1.42)$ & $1.42(1.42)$ \\
\hline Ile & $0.90(0.87)$ & $0.95(0.87)$ & $0.93(0.87)$ & $0.94(0.87)$ & $0.91(0.87)$ & $0.93(0.87)$ \\
\hline Leu & $1.61(1.61)$ & $1.63(1.61)$ & $1.60(1.61)$ & $1.60(1.61)$ & $1.53(1.61)$ & $1.60(1.61)$ \\
\hline Met & $0.50(0.50)$ & $0.46(0.50)$ & $0.49(0.50)$ & $0.47(0.50)$ & $0.49(0.50)$ & $0.49(0.50)$ \\
\hline Met and Cys & $0.82(0.82)$ & $0.78(0.82)$ & $0.81(0.82)$ & $0.78(0.82)$ & $0.80(0.82)$ & $0.81(0.82)$ \\
\hline Thr & $0.95(0.93)$ & $0.97(0.93)$ & $0.95(0.93)$ & $0.94(0.93)$ & $0.94(0.93)$ & $0.95(0.93)$ \\
\hline Trp & $0.22(0.22)$ & $0.23(0.24)$ & $0.24(0.26)$ & $0.27(0.29)$ & $0.30(0.31)$ & $0.30(0.34)$ \\
\hline Val & $1.07(1.02)$ & $1.05(1.02)$ & $1.04(1.02)$ & $1.05(1.02)$ & $1.03(1.02)$ & $1.05(1.02)$ \\
\hline His & $0.47(0.48)$ & $0.46(0.48)$ & $0.46(0.48)$ & $0.45(0.48)$ & $0.44(0.48)$ & $0.46(0.48)$ \\
\hline Phe & $0.92(0.86)$ & $0.92(0.86)$ & $0.91(0.86)$ & $0.90(0.86)$ & $0.88(0.86)$ & $0.89(0.86)$ \\
\hline
\end{tabular}

${ }^{1}$ Diet samples were collected from feeder at the beginning of the trial and on $\mathrm{d} 7$ and 14 . At the end of the trial, samples of each diet were combined, and a composite sample was analyzed for total AA analysis by Ajinomoto Heartland Inc. (Chicago, IL). Samples of the diets were also submitted to Ward Laboratories Inc. (Kearney, NE) for analysis of DM, CP, CF, Ca, P, ash, and crude fat.

${ }^{2}$ Values in parentheses indicate those calculated and are based on values from NRC (1998).

Table 4. Chemical analysis of the diets, Exp. 2 (as-fed basis) ${ }^{1}$

\begin{tabular}{|c|c|c|c|c|c|c|c|}
\hline \multirow[b]{2}{*}{ Item } & \multicolumn{7}{|c|}{ Standardized ileal digestible Trp:Lys ${ }^{2}$} \\
\hline & $14.5 \%$ & $16.5 \%$ & $18.0 \%$ & $19.5 \%$ & $21.0 \%$ & $22.5 \%$ & $24.5 \%$ \\
\hline \multicolumn{8}{|c|}{ Proximate analysis, $\%$} \\
\hline $\mathrm{DM}$ & $90.48(88.26)$ & $90.06(88.27)$ & $90.21(88.27)$ & $90.25(88.27)$ & $90.35(88.27)$ & $89.91(88.27)$ & $89.78(88.28)$ \\
\hline $\mathrm{CP}$ & $19.0(18.1)$ & $19.4(18.2)$ & $18.8(18.2)$ & $18.7(18.2)$ & $18.9(18.2)$ & $19.1(18.2)$ & $18.2(18.2)$ \\
\hline $\mathrm{CF}$ & $3.8(4.2)$ & $3.8(4.2)$ & $4(4.2)$ & $3.9(4.2)$ & $3.5(4.2)$ & $3.8(4.2)$ & $4.0(4.2)$ \\
\hline $\mathrm{Ca}$ & $0.88(0.71)$ & $0.93(0.71)$ & $0.97(0.71)$ & $1.11(0.71)$ & $1.04(0.71)$ & $1.10(0.71)$ & $1.25(0.71)$ \\
\hline $\mathrm{P}$ & $0.52(0.49)$ & $0.52(0.49)$ & $0.55(0.49)$ & $0.54(0.49)$ & $0.52(0.49)$ & $0.53(0.49)$ & $0.54(0.49)$ \\
\hline Fat & $4.8(5.2)$ & $4.7(5.2)$ & $4.9(5.2)$ & $4.9(5.2)$ & $4.7(5.2)$ & $4.7(5.2)$ & $4.7(5.2)$ \\
\hline Ash & $4.89(4.73)$ & $4.75(4.73)$ & $4.82(4.73)$ & $5.39(4.72)$ & $5.35(4.72)$ & $5.18(4.72)$ & $5.57(4.72)$ \\
\hline \multicolumn{8}{|l|}{ AA, $\%$} \\
\hline Lys & $1.19(1.13)$ & $1.18(1.13)$ & $1.22(1.13)$ & $1.22(1.13)$ & $1.17(1.13)$ & $1.16(1.13)$ & $1.19(1.13)$ \\
\hline Ile & $0.73(0.65)$ & $0.75(0.65)$ & $0.75(0.65)$ & $0.75(0.65)$ & $0.74(0.65)$ & $0.76(0.65)$ & $0.77(0.65)$ \\
\hline Leu & $1.82(1.74)$ & $1.86(1.74)$ & $1.86(1.74)$ & $1.87(1.74)$ & $1.85(1.74)$ & $1.90(1.74)$ & $1.89(1.74)$ \\
\hline Met & $0.40(0.39)$ & $0.39(0.39)$ & $0.40(0.39)$ & $0.40(0.39)$ & $0.40(0.39)$ & $0.39(0.39)$ & $0.40(0.39)$ \\
\hline Met and Cys & $0.70(0.70)$ & $0.71(0.70)$ & $0.72(0.70)$ & $0.71(0.70)$ & $0.72(0.70)$ & $0.72(0.70)$ & $0.73(0.70)$ \\
\hline Thr & $0.82(0.78)$ & $0.81(0.78)$ & $0.83(0.78)$ & $0.81(0.78)$ & $0.83(0.78)$ & $0.80(0.77)$ & $0.81(0.77)$ \\
\hline Trp & $0.19(0.17)$ & $0.19(0.19)$ & $0.19(0.21)$ & $0.20(0.22)$ & $0.23(0.24)$ & $0.23(0.25)$ & $0.24(0.27)$ \\
\hline Val & $0.93(0.83)$ & $0.96(0.83)$ & $0.96(0.83)$ & $0.96(0.83)$ & $0.95(0.83)$ & $0.96(0.83)$ & $0.96(0.83)$ \\
\hline His & $0.47(0.44)$ & $0.48(0.44)$ & $0.48(0.44)$ & $0.48(0.44)$ & $0.47(0.44)$ & $0.49(0.44)$ & $0.49(0.44)$ \\
\hline Phe & $0.88(0.81)$ & $0.91(0.81)$ & $0.90(0.81)$ & $0.91(0.81)$ & $0.90(0.81)$ & $0.93(0.81)$ & $0.93(0.81)$ \\
\hline
\end{tabular}

${ }^{1}$ Diet samples were taken from 6 feeders per dietary treatment $3 \mathrm{~d}$ after the beginning of the trial and $3 \mathrm{~d}$ before the end of the trial and were stored at $-20^{\circ} \mathrm{C}$; then $\mathrm{CP}$ and AA analysis was conducted on composite samples by Ajinomoto Heartland Inc. (Chicago, IL). Samples of the diets were also submitted to Ward Laboratories Inc. (Kearney, NE) for analysis of DM, CF, Ca, P, ash, and crude fat.

${ }^{2}$ Values in parentheses indicate those calculated and are based on values from NRC (2012), with the exception of CP and total AA content from corn, soybean meal, and dried distillers grains with solubles, which were analyzed before diet formulation by Ajinomoto Heartland Inc. (Chicago, IL). 
Table 5. Least squares mean estimates $( \pm \mathrm{SEM})$ for growth performance of 6- to 10-kg nursery pigs fed dietary treatments consisting of standardized ileal digestible (SID) Trp:Lys ratios ranging from $14.7 \%$ to $24.0 \%$ (Exp. 1$)^{1}$

\begin{tabular}{lcccccccc}
\hline \hline & \multicolumn{9}{c}{ SID Trp:Lys } & \multicolumn{2}{c}{ Probability P } \\
\cline { 2 - 8 } Item & $14.7 \%$ & $16.5 \%$ & $18.4 \%$ & $20.3 \%$ & $22.1 \%$ & $24.0 \%$ & Linear & Quadratic \\
\hline ADG, g & $226 \pm 14.1$ & $244 \pm 8.5$ & $244 \pm 8.5$ & $266 \pm 14.1$ & $258 \pm 14.1$ & $260 \pm 8.5$ & $<0.022$ & $<0.484$ \\
ADFI, g & $325 \pm 11.6$ & $342 \pm 11.6$ & $342 \pm 11.6$ & $349 \pm 11.6$ & $341 \pm 11.6$ & $363 \pm 11.6$ & $<0.057$ & $<0.939$ \\
G:F & $0.718 \pm 0.011$ & $0.697 \pm 0.011$ & $0.694 \pm 0.011$ & $0.750 \pm 0.011$ & $0.751 \pm 0.011$ & $0.716 \pm 0.011$ & $<0.012$ & $<0.500$ \\
BW, kg & & & & & & & \\
$\quad$ & & & & & & & \\
$\quad$ d 0 & $9.3 \pm 0.06$ & $6.3 \pm 0.06$ & $6.2 \pm 0.06$ & $6.3 \pm 0.06$ & $6.2 \pm 0.06$ & $6.3 \pm 0.06$ & $<0.753$ & $<0.870$ \\
$\quad$ d 14 & $9.4 \pm 0.19$ & $9.7 \pm 0.19$ & $9.7 \pm 0.09$ & $10.0 \pm 0.19$ & $9.9 \pm 0.19$ & $9.9 \pm 0.19$ & $<0.052$ & $<0.294$ \\
SID Trp intake, g/d & $0.621 \pm 0.029$ & $0.736 \pm 0.029$ & $0.818 \pm 0.029$ & $0.917 \pm 0.029$ & $0.978 \pm 0.029$ & $1.132 \pm 0.029$ & $<0.001$ & $<0.687$ \\
SID Trp g/kg gain & $2.8 \pm 0.11$ & $3.0 \pm 0.06$ & $3.4 \pm 0.06$ & $3.5 \pm 0.11$ & $3.8 \pm 0.11$ & $4.4 \pm 0.11$ & $<0.001$ & $<0.075$ \\
\hline
\end{tabular}

${ }^{1}$ A total of 255 nursery pigs (PIC $327 \times 1050$, initially $6.3 \mathrm{~kg}$ and $3 \mathrm{~d}$ postweaning) were used in a 14-d trial with 6 to 7 pigs per pen and 7 pens per treatment. Diets were formulated to $1.30 \%$ SID Lys based on data of Nemechek et al. (2011). The SID Trp intake and gram per kilogram gain were evaluated using diet calculated values for Trp.

whereby the SID Trp:Lys explanatory variable is expressed as a proportion (i.e., 0.180) rather than a percentage (i.e., 18.0\%) for numerical stability. Based on the best-fitting BLL model, the estimated minimum SID Trp:Lys requirement to achieve maximum G:F was $20.4 \%$ (95\% CI: [14.3\%, 26.5\%]).

To the author's knowledge, this is the first attempt to try to quantify uncertainty around the SID Trp:Lys ratio requirement. Therefore, after this first experiment, a subsequent study was conducted on a larger scale in a commercial facility with a 7-point titration and slightly wider treatment ranges to reduce the uncertainty around the estimates and evaluate the response to increasing SID Trp:Lys ratios.

In Exp. 2 (11 to $20 \mathrm{~kg} \mathrm{BW}$ ), increasing SID Trp:Lys ratio quadratically increased (Table 6) ADG $(P=0.001)$, ADFI $(P=0.006), \mathrm{G}: \mathrm{F}(P=0.002)$, final BW $(P=0.001)$, grams of SID Trp intake per day $(P=0.026)$, and grams of SID Trp intake per kilogram of gain $(P=0.006)$.

For ADG in the 11- to 20-kg BW pigs (Exp. 2), the best-fitting model was a QP (BIC: 198.1) compared with BLL and BLQ models (BIC: 204.8 and 204.8, re-

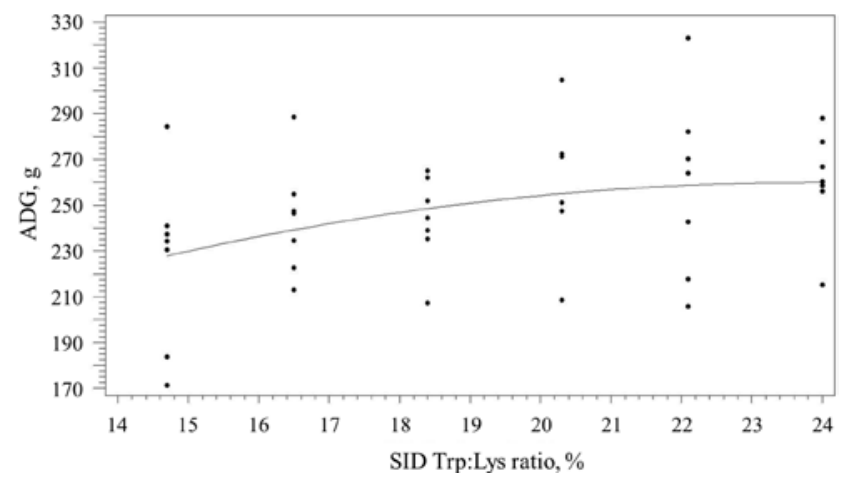

Figure 1. Fitted quadratic polynomial regression model on ADG as a function of increasing standardized ileal digestible (SID) Trp:Lys in 6- to $10-\mathrm{kg}$ pigs (Exp. 1.). The maximum ADG was estimated at $23.9 \%(95 \%$ confidence interval: $[<14.5 \%,>24.5 \%])$ SID Trp:Lys. spectively). The estimated regression equation for the best-fitting QP model (Fig. 3) was

$$
\begin{aligned}
& A D G=-317+7,259 \times(\text { Trp:Lys })-17,110 \times \\
& (\text { Trp:Lys })^{2} .
\end{aligned}
$$

On the basis of the best-fitting QP model, the maximum ADG was estimated at $21.2 \%$ (95\% CI: $20.5 \%$ to 21.9\%) SID Trp:Lys. Note the reduced uncertainty (i.e., narrower CI) around the estimated Trp:Lys ratio requirement for maximum ADG compared with that in Exp 1.

For G:F in the 11- to 20-kg BW pigs (Exp. 2), BLL and BLQ models had comparable fits (BIC: 346.1 and 346.1, respectively), whereas QP showed a less adequate fit (BIC: 355.2). The comparable fit of these models may be explained by the scarcity of information on G:F for the range of SID Trp:Lys ratios for which the functional relationship is estimated; that is, G:F observations were available for only 2 SID Trp:Lys ratios before the estimated plateau in G:F was detected. Note that the same number of unknown fixed-effect parameters occur in the BLL model (i.e., $L_{B L L}, U_{l}$, and $R_{B L L}$ ) and the BLQ model (i.e., $L_{B L Q}, U_{q}$, and $R_{B L Q}$ ), so the

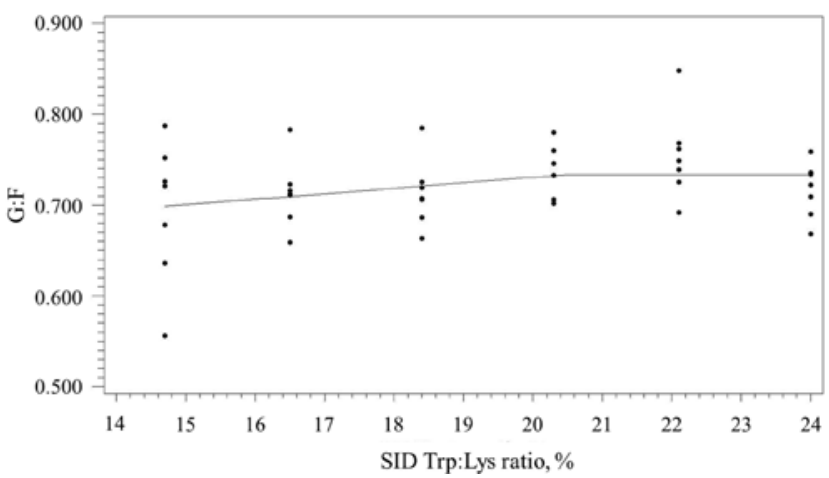

Figure 2. Fitted broken-line linear regression model on G:F as a function of increasing standardized ileal digestible (SID) Trp:Lys in 6- to $10-\mathrm{kg}$ pigs (Exp. 1). The maximum G:F was estimated at 20.4\% $(95 \%$ confidence interval: $[14.3 \%, 26.5 \%])$. 


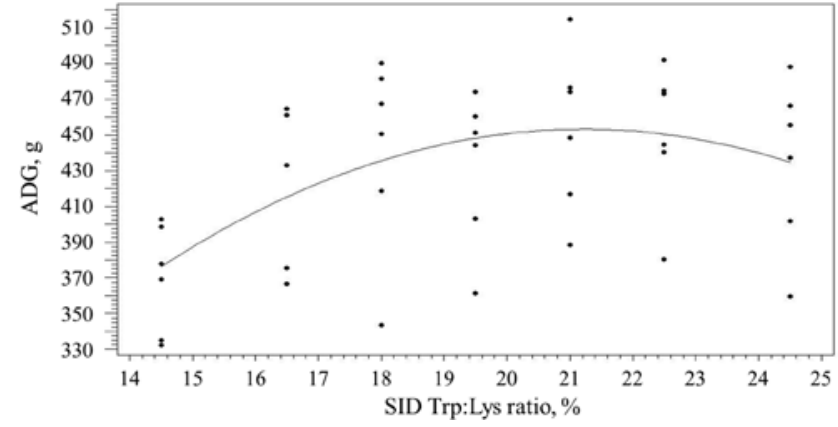

Figure 3. Fitted quadratic polynomial regression model on ADG as a function of increasing standardized ileal digestible (SID) Trp:Lys in 11- to $20-\mathrm{kg}$ pigs (Exp. 2.). The maximum ADG was estimated at $21.2 \%(95 \%$ confidence interval: [20.5\%, 21.9\%]) SID Trp:Lys.

principle of parsimony favoring simpler models would, in this case, not contribute to model selection. Taken together, these issues can help explain, at least partially, the impaired discrimination in differential fit between BLL and BLQ models. On the basis of the best-fitting BLL and BLQ models, alternative estimated regression equations (Fig. 4) were

$$
\begin{array}{r}
\mathrm{G}: \mathrm{F}=0.5844-1.95(0.166-\text { Trp:Lys }) \\
\text { if SID Trp:Lys }<16.6 \%,
\end{array}
$$

$$
\mathrm{G}: \mathrm{F}=0.5844 \text { if SID Trp:Lys } \geq 16.6 \%
$$

for the BLL model and

$$
\begin{array}{r}
\mathrm{G}: \mathrm{F}=0.5844-59.80(0.171-\text { Trp:Lys })^{2} \\
\text { if SID Trp:Lys }<17.1 \%,
\end{array}
$$

$\mathrm{G}: \mathrm{F}=0.5844$ if SID Trp:Lys $\geq 17.1 \%$

for the BLQ model.

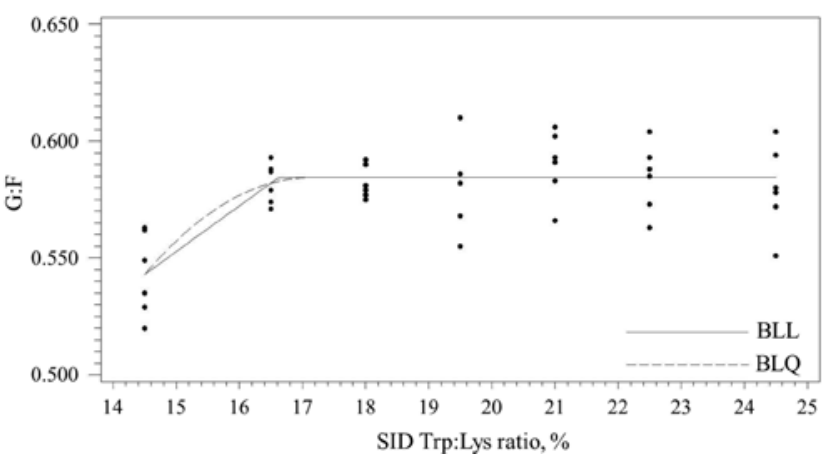

Figure 4. Fitted broken-line linear (BLL) and broken-line quadratic (BLQ) regression models on G:F as a function of increasing standardized ileal digestible (SID) Trp:Lys in 11- to 20-kg pigs (Exp. 2.). The maximum G:F was estimated at $16.6 \%(95 \%$ confidence interval [CI]: [16.0\%, $17.3 \%])$ and $17.1 \%(95 \%$ CI: $[16.6 \%, 17.7 \%])$ SID Trp:Lys in the BLL and BLQ models, respectively.

The estimated SID Trp:Lys requirements for G:F were $16.6 \%$ (95\% CI: 16.0 to 17.3$)$ and $17.1 \%(95 \%$ CI: 16.6 to 17.7$)$ based on the BLL and BLQ models, respectively. Again, similar to the estimated SID Trp:Lys requirements for maximum ADG, the CI for SID Trp:Lys requirements for maximum G:F was narrower than that in Exp. 1.

Broken-line models and QP models can be used (through inverse prediction) to estimate the minimum level of requirement that maximizes average growth performance in the intended swine population. From a conceptual standpoint, both types of models may address the question of interest, but finer differences between the fit of the models become apparent when describing the relationship between growth performance and SID Trp:Lys. This illustrates the importance of objectively selecting a model based on its fit to the data (Littell et al., 2006). When developing requirement curves, the coefficient of determination $R^{2}$ traditionally

\begin{tabular}{|c|c|c|c|c|c|c|c|c|c|}
\hline \multirow[b]{2}{*}{ Item } & \multicolumn{7}{|c|}{ SID Trp:Lys } & \multicolumn{2}{|c|}{ Probability, $P$} \\
\hline & $14.5 \%$ & $16.5 \%$ & $18.0 \%$ & $19.5 \%$ & $21.0 \%$ & $22.5 \%$ & $24.5 \%$ & Linear & Quadratic \\
\hline \multicolumn{10}{|l|}{ d 0 to 21} \\
\hline ADG, $g$ & $369 \pm 20.2$ & $428 \pm 20.2$ & $442 \pm 20.2$ & $432 \pm 20.2$ & $453 \pm 17.6$ & $451 \pm 17.6$ & $435 \pm 17.6$ & $<0.001$ & $<0.001$ \\
\hline ADFI, $g$ & $682 \pm 35.2$ & $735 \pm 31.1$ & $759 \pm 35.2$ & $749 \pm 35.2$ & $768 \pm 31.1$ & $773 \pm 31.1$ & $750 \pm 31.1$ & $<0.001$ & $<0.006$ \\
\hline $\mathrm{G}: \mathrm{F}$ & $0.543 \pm 0.008$ & $0.582 \pm 0.005$ & $0.582 \pm 0.005$ & $0.578 \pm 0.008$ & $0.590 \pm 0.005$ & $0.584 \pm 0.005$ & $0.580 \pm 0.008$ & $<0.002$ & $<0.002$ \\
\hline \multicolumn{10}{|l|}{$\mathrm{BW}, \mathrm{kg}$} \\
\hline $\mathrm{d} 0$ & $11.3 \pm 0.55$ & $11.3 \pm 0.55$ & $11.2 \pm 0.55$ & $11.2 \pm 0.55$ & $11.2 \pm 0.55$ & $11.3 \pm 0.55$ & $11.2 \pm 0.55$ & $<0.844$ & $<0.952$ \\
\hline $\mathrm{d} 21$ & $19.0 \pm 0.87$ & $20.2 \pm 0.87$ & $20.7 \pm 0.87$ & $20.3 \pm 0.94$ & $20.8 \pm 0.87$ & $20.7 \pm 0.87$ & $20.4 \pm 0.87$ & $<0.001$ & $<0.001$ \\
\hline SID Trp intake, g/d & $0.959 \pm 0.069$ & $1.176 \pm 0.063$ & $1.325 \pm 0.069$ & $1.417 \pm 0.069$ & $1.564 \pm 0.063$ & $1.686 \pm 0.063$ & $1.783 \pm 0.063$ & $<0.001$ & $<0.026$ \\
\hline SID Trp, g/kg gain & $2.6 \pm 0.04$ & $2.8 \pm 0.02$ & $3.0 \pm 0.02$ & $3.3 \pm 0.04$ & $3.5 \pm 0.04$ & $3.7 \pm 0.04$ & $4.1 \pm 0.04$ & $<0.001$ & $<0.006$ \\
\hline
\end{tabular}
has been used as the primary indicator of model fit to select among competing models (Pesti et al., 2009).

Table 6. Least squares mean estimates ( $( \pm \mathrm{SEM})$ for growth performance of 11 - to $20-\mathrm{kg}$ nursery pigs fed dietary treatments of standardized ileal digestible (SID) Trp:Lys ratio ranging from $14.5 \%$ to $24.5 \%$ (Exp. 2$)^{1}$

\footnotetext{
${ }^{1} \mathrm{~A}$ total of 1,088 pigs (PIC $337 \times 1050$, initially $11.2 \mathrm{~kg} \mathrm{BW}$ and $28 \mathrm{~d}$ postweaning) were used in a 21 -d growth trial with 24 to 27 pigs per pen and 6 pens per treatment. The NRC (2012) model was used to determine the Lys requirement of mixed-gender pens of pigs at the end of the BW range (22.7 kg), and that value was reduced by $0.10 \%$. The SID Trp intake and gram per kilogram gain were evaluated using diet calculated values for Trp.
} 
However, within a mixed-model framework as in this study, the calculation and interpretation of $R^{2}$ is fraught with ambiguities. First, $R^{2}$ is not uniquely defined when multiple sources of random variability are present in the data (Schabenberger and Pierce, 2002). Such multiple definitions of $R^{2}$ in the mixed-model framework impair the arguably intuitive interpretation of $R^{2}$ as a proportion of variability explained by $\mathrm{X}$ that has been so appealing in the animal sciences. Furthermore, attempts to calculate $R^{2}$ in the mixed-model framework fail to take into account model complexity in the presence of random effects (Kvalseth, 1985). In turn, other information criteria that take into consideration the design structure of the experiment are available for model selection in the mixed-model framework. These include, but are not limited to, the maximum likelihood-based Akaike information criterion and Schwarz's BIC (Milliken and Johnson, 2009). Schwarz's BIC is often used in mixed models, and its calculation is slightly more conservative than the AIC and tends to favor more parsimonious models (Schwarz, 1978); for this reason BIC was the primary indicator of model fit when assessing the competing dose response models. A 2-point difference in BIC is usually considered to be indicative of improved fit of the model with lower BIC (Raftery, 1996). The results indicate that for ADG in both early and late nursery pigs, the QP model was the single best fitting model. In contrast, for G:F the BLL was the best-fitting model in early nursery pigs. In late nursery pigs, the BLL and BLQ models had comparable BIC, indicating comparable fits to the data, so the regression equations for both models were provided. Although the CI for SID Trp:Lys ratio requirements overlap for the BLL and BLQ models, the functional forms assumed by the models induced a point estimate for BLQ that was numerically slightly higher than that of the BLL model.

In addition to considering biologic performance, nutritionists should also take into account economic considerations during diet formulation. It is generally economically unfeasible to formulate diets that meet requirements to achieve $100 \%$ of the maximized average performance, so arbitrary target levels of average performance (i.e., 95\%) are commonly used in the industry (Pesti et al., 2009). Therefore, the current study also provides fitted prediction equations that can be used to estimate ADG and G:F on the basis of different SID Trp:Lys requirements. The SID Trp:Lys ratios needed to achieve different target average ADG and G:F levels for nursery pigs are demonstrated in Table 7 . An approximately 18\% SID Trp:Lys ratio would be needed to achieve 95\% of the maximum ADG and $98 \%$ of the maximum G:F in Exp. 1, whereas the same ratio would achieve $96 \%$ of the maximum ADG and $100 \%$ of the maximum G:F in Exp. 2. In early and late nursery pigs, $99 \%$ of the
Table 7. Standardized ileal digestible (SID) Trp:Lys ratio at different target performance levels of nursery pigs ${ }^{1}$

\begin{tabular}{|c|c|c|c|c|c|c|}
\hline \multirow[b]{2}{*}{ Item } & \multicolumn{6}{|c|}{ Percentage of maximum performance } \\
\hline & $95 \%$ & $96 \%$ & $97 \%$ & $98 \%$ & $99 \%$ & $100 \%$ \\
\hline \multicolumn{7}{|c|}{ Exp. 1 (6- to 10-kg BW pigs) } \\
\hline \multicolumn{7}{|l|}{$\mathrm{ADG}$} \\
\hline QP & 18.1 & 18.7 & 19.4 & 20.2 & 21.3 & 23.9 \\
\hline \multicolumn{7}{|l|}{$\mathrm{G}: \mathrm{F}$} \\
\hline BLL & 14.3 & 15.5 & 16.7 & 18.0 & 19.2 & 20.4 \\
\hline \multicolumn{7}{|c|}{ Exp. 2 (11- to $20-\mathrm{kg}$ BW pigs) } \\
\hline \multicolumn{7}{|l|}{ ADG } \\
\hline QP & 17.6 & 18.0 & 18.4 & 18.9 & 19.6 & 21.2 \\
\hline \multicolumn{7}{|l|}{ G:F } \\
\hline BLL & 15.1 & 15.4 & 15.7 & 16.0 & 16.3 & 16.6 \\
\hline BLQ & 14.9 & 15.1 & 15.4 & 15.7 & 16.1 & 17.1 \\
\hline
\end{tabular}

${ }^{1}$ Derived from equations presented in the text. BLL $=$ broken-line linear; $\mathrm{BLQ}=$ broken-line quadratic; $\mathrm{QP}=$ quadratic polynomial.

maximum ADG was reached at $21.3 \%$ and $19.6 \%$ SID Trp:Lys, respectively. These relative differences in performance can be translated into economic terms on the basis of localized economic conditions while taking into account the underlying biologic performance.

In general, these findings add to the body of literature in which low levels of SID Trp in the diets of pigs reduce ADFI and, consequently, reduce ADG and G:F (Simongiovanni et al., 2012). The absorbed Trp is converted into 5-hydroxytryptophan, which is a metabolite that can cross the blood-brain barrier and work as a precursor for serotonin after being decarboxylated (Le Floc'h et al., 2011). As a result, the inclusion of different levels of Trp in pig diets has been shown to manipulate serotonin-mediated feed intake (Batterham et al., 1994; Simongiovanni et al., 2012). Although the results on SID Trp:Lys requirements for the 6- to 10$\mathrm{kg} \mathrm{BW}$ phase (Exp. 1) were inconclusive, the study for the 11- to 20-kg BW phase (Exp. 2) indicates the SID Trp:Lys requirements for maximum ADG were greater than those for maximum G:F, which is consistent with other studies (Ma et al., 2010; Petersen, 2011). In turn, the NRC (2012) recommended SID Trp:Lys ratio is $16.3 \%$ for nursery pigs, whereas the National Swine Nutrition Guide (2010) recommended SID Trp:Lys at $16.8 \%$ for nursery pigs. It is worth noticing that neither of these 2 standards distinguish between requirements for $\mathrm{ADG}$ and requirements for $\mathrm{G}: \mathrm{F}$; in fact, these standards correspond closely with estimates of SID Trp:Lys requirements for maximum G:F, but they may underestimate requirements for maximum ADG. 


\section{LITERATURE CITED}

AOAC International. 2012. Official methods of analysis of AOAC International. 19th ed. AOAC Int., Gaithersburg, MD.

Batterham, E. S., L. M.Andersen, and D. R. Baigent. 1994. Utilization of ileal digestible amino acids by growing pigs: Tryptophan. Br. J. Nutr. 71:345-360. doi:10.1079/BJN19940143.

Cromwell, G. L., C. C. Calvert, T. R. Cline, J. D. Crenshaw, T. D. Crenshaw, R. A. Easter, R. C. Ewan, C. R. Hamilton, G. M. Hill, A. J. Lewis, D. C. Mahan, E. R. Miller, J. L. Nelssen, J. E. Pettigrew, L. F. Tribble, T. L. Veum, and J. T. Yen. 1999. Variability among sources and laboratories in nutrient analyses of corn and soybean meal. J. Anim. Sci. 77:3262-3273.

Guzik, A. C., M. J. Pettitt, E. Beltranena, L. L. Southern, and B. J. Kerr. 2005. Threonine and tryptophan ratios fed to nursery pigs. J. Anim. Physiol. Anim. Nutr. 89:297-302. doi:10.1111/ j.1439-0396.2005.00529.x.

Kvalseth, T. O. 1985. Cautionary note about $\mathrm{R}^{2}$. Am. Stat. 39:279-285.

Lavagnini, I., and F. Magno. 2006. A statistical overview on univariate calibration, inverse regression, and detection limits: application to gas chromatography/mass spectrometry technique. Mass. Spectrom. Rev. 26:1-18.

Le Floc'h, N., W. Otten, and E. Merlot. 2011. Tryptophan metabolism, from nutrition to potential therapeutic applications. Amino Acids 41:1195-1205. doi:10.1007/s00726-010-0752-7.

Lewis, A. J. 2000. Amino acids in swine nutrition. In: A. J. Lewis and L. L. Southern, editors, Swine nutrition. 2nd ed. CRC Press, Boca Raton, FL. p. 137-139.

Littell, R. C., G. A. Milliken, W. W. Stroup, R. D. Wolfinger, and O. Schabenberger. 2006. SAS $®$ for mixed models. 2nd ed., SAS Inst. Inc., Cary, NC.

Ma, L., Z. P. Zhu, R. B. Hinson, G. L. Allee, J. D. Less, D. D. Hall, H. Yang, and D. P. Holzgraefe. 2010. Determination of SID Trp:Lys ratio requirement of 11- to 22-kg pigs fed diets containing 30\% DDGS. J. Anim. Sci. 88(E-Suppl. 3):151. (Abstr.)

Milliken, G. A., and D. E. Johnson. 2009. Analysis of messy data: Designed experiments. 2nd ed. Vol. 1. CRC Press, Boca Raton, FL.
National Swine Nutrition Guide. 2010. Table of nutrient recommendations, ingredient composition, and usage rates. U.S. Pork Cent. Excell., Ames, IA.

Nemechek, J. E., M. D. Tokach, S. S. Dritz, R. D. Goodband, J. M. DeRouchey, and J. L. Nelssen. 2011. Effects of deleting crystalline amino acids from low-CP, amino acid-fortified diets and dietary valine:lysine ratio for nursery pigs from 6.8 to 11.3 kg. J. Anim. Sci. 89(E-Suppl. 2):97. (Abstr.)

NRC. 1998. Nutrient requirements of swine. 10th rev. ed. Natl. Acad. Press, Washington, DC.

NRC. 2012. Nutrient requirements of swine. 11th rev. ed. Natl. Acad. Press, Washington, DC.

Pesti, G. M., D. Vedenov, J. A. Cason, and L. Billard. 2009. A comparison of methods to estimate nutritional requirements from experimental data. Br. Poult. Sci. 50:16-32. doi:10.1080/00071660802530639.

Petersen, G. I. 2011. Estimation of the ideal standardized ileal digestible tryptophan:lysine ratio in 10 to $20 \mathrm{~kg}$ pigs. $\mathrm{PhD}$ Diss. Univ. of Illinois, Urbana-Champaign.

Raftery, A. E. 1996. Approximate Bayes factors and accounting for model uncertainty in generalized linear regression models. Biometrika 83:251-266. doi:10.1093/biomet/83.2.251.

Robbins, K. R., A. M. Saxton, and L. L. Southern. 2006. Estimation of nutrient requirements using broken-line regression analysis. J. Anim. Sci. 84:E155-E165.

Schabenberger, O., and F. J. Pierce. 2002. Contemporary statistical models for the plant and soil sciences. CRC Press, Boca Raton, FL.

Schwarz, G. 1978. Estimating dimension of a model. Ann. Stat. 6:461-464. doi:10.1214/aos/1176344136.

Simongiovanni, A., E. Corrent, N. Le Floc'h, and J. van Milgen. 2012. Estimation of the tryptophan requirement in piglets by meta-analysis. Animal 6:594-602. doi:10.1017/ S1751731111001960.

Stein, H. H., M. F. Fuller, P. J. Moughan, B. Sève, R. Mosenthin, A. J. M. Jansman, J. A. Fernández, and C. F. M. de Lange. 2007. Definition of apparent, true, and standardized ileal digestibility of amino acids in pigs. Livest. Sci. 109:282-285. doi:10.1016/j.livsci.2007.01.019. 
Reproduced with permission of the copyright owner. Further reproduction prohibited without permission. 